\title{
Urinary MicroRNA Profiling in the Nephropathy of Type 1 Diabetes
}

\author{
Christos Argyropoulos ${ }^{19}$, Kai Wang ${ }^{29}$, Sara McClarty ${ }^{2}$, David Huang ${ }^{2,3}$, Jose Bernardo ${ }^{1}$, Demetrius Ellis ${ }^{5}$, \\ Trevor Orchard ${ }^{4}$, David Galas ${ }^{3,5,6}$, John Johnson ${ }^{1 *}$
}

1 Renal and Electrolyte Division, Department of Medicine, University of Pittsburgh, Pittsburgh, Pennsylvania, United States of America, 2 Institute for Systems Biology, Seattle, Washington, United States of America, 3 Luxembourg Center for Systems Biomedicine, University of Luxembourg, Esch-sur-Alzette, Luxembourg, 4 Department of Epidemiology, Graduate School of Public Health, University of Pittsburgh, Pittsburgh, Pennsylvania, United States of America, $\mathbf{5}$ Children's Hospital of Pittsburgh, Pittsburgh, Pennsylvania, United States of America, 6 Pacific Nothwest Diabetes Research Institute, Seattle, Washington, United States of America

\begin{abstract}
Background: Patients with Type 1 Diabetes (T1D) are particularly vulnerable to development of Diabetic nephropathy (DN) leading to End Stage Renal Disease. Hence a better understanding of the factors affecting kidney disease progression in T1D is urgently needed. In recent years microRNAs have emerged as important post-transcriptional regulators of gene expression in many different health conditions. We hypothesized that urinary microRNA profile of patients will differ in the different stages of diabetic renal disease.

Methods and Findings: We studied urine microRNA profiles with qPCR in 40 T1D with $>20$ year follow up 10 who never developed renal disease $(\mathrm{N})$ matched against 10 patients who went on to develop overt nephropathy (DN), 10 patients with intermittent microalbuminuria (IMA) matched against 10 patients with persistent (PMA) microalbuminuria. A Bayesian procedure was used to normalize and convert raw signals to expression ratios. We applied formal statistical techniques to translate fold changes to profiles of microRNA targets which were then used to make inferences about biological pathways in the Gene Ontology and REACTOME structured vocabularies. A total of 27 microRNAs were found to be present at significantly different levels in different stages of untreated nephropathy. These microRNAs mapped to overlapping pathways pertaining to growth factor signaling and renal fibrosis known to be targeted in diabetic kidney disease.

Conclusions: Urinary microRNA profiles differ across the different stages of diabetic nephropathy. Previous work using experimental, clinical chemistry or biopsy samples has demonstrated differential expression of many of these microRNAs in a variety of chronic renal conditions and diabetes. Combining expression ratios of microRNAs with formal inferences about their predicted mRNA targets and associated biological pathways may yield useful markers for early diagnosis and risk stratification of DN in T1D by inferring the alteration of renal molecular processes.
\end{abstract}

Citation: Argyropoulos C, Wang K, McClarty S, Huang D, Bernardo J, et al. (2013) Urinary MicroRNA Profiling in the Nephropathy of Type 1 Diabetes. PLoS ONE 8(1): e54662. doi:10.1371/journal.pone.0054662

Editor: Karin Jandeleit-Dahm, Baker IDI Heart and Diabetes Institute, Australia

Received June 29, 2012; Accepted December 17, 2012; Published January 24, 2013

Copyright: (C) 2013 Argyropoulos et al. This is an open-access article distributed under the terms of the Creative Commons Attribution License, which permits unrestricted use, distribution, and reproduction in any medium, provided the original author and source are credited.

Funding: This research was supported by NIH grant \#DK34818 and the ISB University of Luxemburg program. The funders had no role in study design, data collection and analysis, decision to publish, or preparation of the manuscript.

Competing Interests: While engaged in this project, Dr. Christos Argyropoulos was a salaried employee of the University of Pittsburgh. Since the completion of the work presented herein Dr. Argyropoulos has served as a consultant to the Medical Department of the Greek Affiliate of Abbott Laboratories, a global healthcare company that manufactures diabetes care products and develops pharmaceuticals for the treatment of diabetic nephropathy. The views and opinions in this research project are solely those of the contributing authors and do not necessarily reflect those of Abbott Laboratories.

*E-mail: johnsonj@pitt.edu

9 These authors contributed equally to this work.

\section{Introduction}

Diabetic nephropathy (DN) is the leading cause of End Stage Renal Disease (ESRD) in the Western world, accounting for more than $40 \%$ of cases. Patients with either type 1 (T1D) or 2 (T2D) diabetes are at risk of $\mathrm{DN}$, but the disease burden is higher in the former group [1]. Hence a better understanding of the factors affecting disease progression(2) from hyperfiltration to microalbuminuria(MA), dipstick positive macroalbuminuria, impaired filtration and ESRD in patients with T1D is urgently needed. The molecular pathophysiology [2] of diabetic nephropathy is multifactorial, involving hemodynamic factors (Vascular Endothelial Growth Factor, renin-angiotensin-aldosterone and endothelin systems), proinflammatory (e.g. Interleykin IL-1, 6,18) and profibrotic cytokines (such as Transforming Growth Factor beta, TGF $\beta$ ) as well as other biochemical derangements (polyol, Protein Kinase G). Nevertheless, the manner in which these diverse molecular processes are regulated, resulting in distinct clinical courses of individual patients remains poorly defined.

In recent years microRNAs (miRNAs), a family of short (average of 22nt long), naturally occurring, small antisense noncoding RNAs have emerged as important post-transcriptional regulators of gene expression (see review [3]). First described in $C$. elegans [4], they have since been discovered to be widely distributed, endogenous controllers of gene and protein expression by binding to the 3 '-untranslated region of specific mRNAs and 
interfering with protein synthesis by inducing mRNA degradation or repressing translation $[5,6]$. A number of these miRNAs have also been identified in the extracellular environment. As they may regulate a significant portion of the transcriptome and proteome, considerable attention has focused on miRNAs as mediators or biomarkers of illness. Previous work in diabetic renal disease (see reviews [7-10]) performed in cell cultures, animal models or formalin fixed human biopsy material has linked a number of miRNAs to the development of nephropathy. Nevertheless, there have been no comprehensive studies examining miRNA signatures in human urine in relation to either longitudinal clinical outcomes and/or the level of urinary albumin, which is the current gold standard for detecting and staging diabetic nephropathy in the clinic. The goal of this pilot study is to identify the differences on urinary miRNA profiles in patients with long standing T1D who were either free from diabetic nephropathy or had developed variable degrees of albuminuria after long follow-up. In addition, by integrating experimentally verified alterations on urinary miRNAs with miRNA target prediction databases, we translated the changes of miRNAs into hypotheses about signalling pathways associated with nephropathy induced by diabetes.

\section{Materials and Methods}

\section{Patients and Samples}

Urine samples from participants in the Pittsburgh Epidemiology of Diabetes Complications (EDC) study were examined. The EDC study is a historical prospective cohort which recruited patients from Children's University Hospital of Pittsburgh Registry of all cases of T1D, diagnosed or seen within a year of diagnosis between January $1^{\text {st }} 1950$ and May $31^{\text {st }} 1980$. Participants were followed thereafter with repeat exams biennially for 10 years and again at 18 years. Follow up of all participants in the EDC was censored for this analysis on December $31^{\text {st }} 2000$.

In the EDC, diabetic renal disease was characterized in terms of its progression from a normoalbuminuric urine examination to progressively higher amounts of albumin in the urine (microalbuminuria) to overt nephropathy. Microalbuminuria was defined as $20-200 \mu \mathrm{g} / \mathrm{min}$ in at least two of three timed urines (24hr, overnight, and $4 \mathrm{hr}$ clinic visit) and was further classified as intermittent(IMA) or persistent (PMA) on the basis of subsequently reverting to normoalbuminuria or persisting at least to microalbuminuria level throughout further follow up respectively. Diabetic nephropathy was defined as an albumin excretion rate $>200 \mu \mathrm{g} / \mathrm{min}$ in at least two of three timed urine collections (24-h, overnight, and post-clinic). In the absence of urine, a serum creatinine $>2 \mathrm{mg} / \mathrm{dl}$ or renal failure was accepted as an alternative diagnostic criterion for overt nephropathy. For the purpose of this report we analyzed urine from matched samples of a) diabetic patients who never developed microalbuminuria or nephropathy after prolonged (25 year) follow - up vs. those with DN and b) patients who developed IMA matched against EDC participants who developed PMA. In the case of the $\mathrm{N} v \mathrm{DN}$ group we collected a single urine sample, while two samples were analyzed from patients who developed microalbuminuria : a (baseline)urine sample from the last visit which tested negative for albumin and the subsequent (follow-up, albuminuric) sample which was collected 2 years after the first. Matching in the 2 sample sets was independently carried out on the basis of age, sex, duration of disease and levels of Hemoglobin Alc (HBAlc) to account for unmeasured confounders.

\section{RNA Isolation}

The RNA from urine was isolated using the miRNeasy kit (Qiagen, Germantown, MD). In brief, $700 \mu \mathrm{l}$ of QIAzol reagent was added to $200 \mu \mathrm{l}$ of urine sample. The sample was mixed in a tube followed by adding $140 \mu \mathrm{l}$ of chloroform. After mixing vigorously for 15 seconds, the sample was then centrifuged at $12,000 \times \mathrm{g}$ for 15 minutes at $4^{\circ} \mathrm{C}$. The upper aqueous phase was carefully transferred to a new collection tube, and 1.5 volume of ethanol containing binding buffer from the kit was added and mixed. The sample was then applied directly to a silica membrane containing column and the RNA was retained and cleaned by using buffers provided in the kit. The immobilized cleaned RNA was then eluted from the membrane into a collection tube with a low salt elution buffer or water. The quality and quantity of the RNA was evaluated by $260 / 280$ ratio and Agilent 2100 Bioanalyzer (Agilent Technologies, Santa Clara CA).

\section{miRNA Profiling}

In brief, the cDNA was generated from $20 \mu \mathrm{l}$ of RNA using buffer and enzyme provide in the Qiagen kit. After incubating the cDNA synthesis reaction at $42^{\circ} \mathrm{C}$ for 60 minutes, the cDNA was diluted to $8 \mathrm{ml}$ with $\mathrm{SYBR}$ containing PCR reagents from Exiqon and water. The plates were then loaded onto ABI 7900HT real-time PCR system and the threshold cycle $\left(\mathbf{C}_{t}\right)$ was measured with standard methods. Exiqon miRNA qPCR panels 1 and 2 (Version 1) were used, that included probes for 748 unique miRNA. Each miRNA species was assayed once per panel with the exception of miR-423-5p, miR-103, miR-191 and the three non-coding RNA species U6, SNORD38B and SNORD39A for which duplicate reactions was set up as per panel manufacturer instructions. Although suggested as reference genes (biological controls) by the panel manufacturer the 6 microRNAs/small nuclear RNAs were not used as referents during normalization. Nevertheless their presence in multiple technical replicates in any given panel, allowed us to derive panel specific normalization factors which were applied to the raw expression levels of all microRNAs. A single inter-plate calibrator spiked in control (UniSP3) was run 6 times per plate and was used to normalize the expression levels of all miRNAs included in each of the qPCR panels. A second spiked in control (UniSP6) was included in some but not all urine reactions as a dual positive negative control and was thus not considered in subsequent analyses (including normalization). We also included a notemplate negative control in all assays (nine replicates per assay) as per manufacture guidelines. To resolve discrepancies in the nomenclature of miRNA species, we mapped names of miRNAs present in the Exiqon plates to the most current ones in miRBase (version 18, November 2011) and the associated MIMAT accession numbers (Table $\mathrm{S} 1$ ).

\section{Statistical Analyses}

Quantification cycle (Threshold Crossing) $\mathbf{G}_{\mathbf{q}}$ visualization, signal analysis and normalization. In order to classify individual patient samples and visualize the resemblance in the corresponding profiles we applied Principal Component Analysis (PCA) to the corrected $\mathrm{C}_{\mathrm{q}}$ values obtained from the raw $\mathrm{C}_{\mathrm{q}}$ measurements after subtracting the quantification cycle number of the spiked-in control. To handle missing data in the expression of miRNAs across samples we applied a specific variety of PCA, i.e. Probabilistic PCA (PPCA) [11] that combines the Expectation Maximization (EM) with PCA to simultaneous estimate missing expression values and the principal components in the dataset. Results of PPCA were plotted as bivariate 


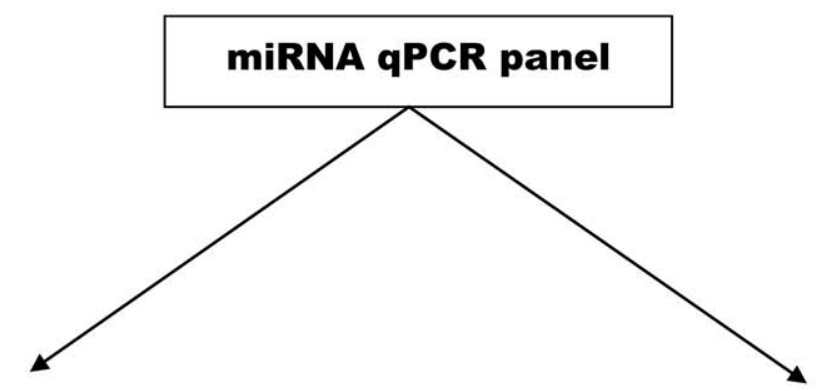

\section{Replicate Reactions}

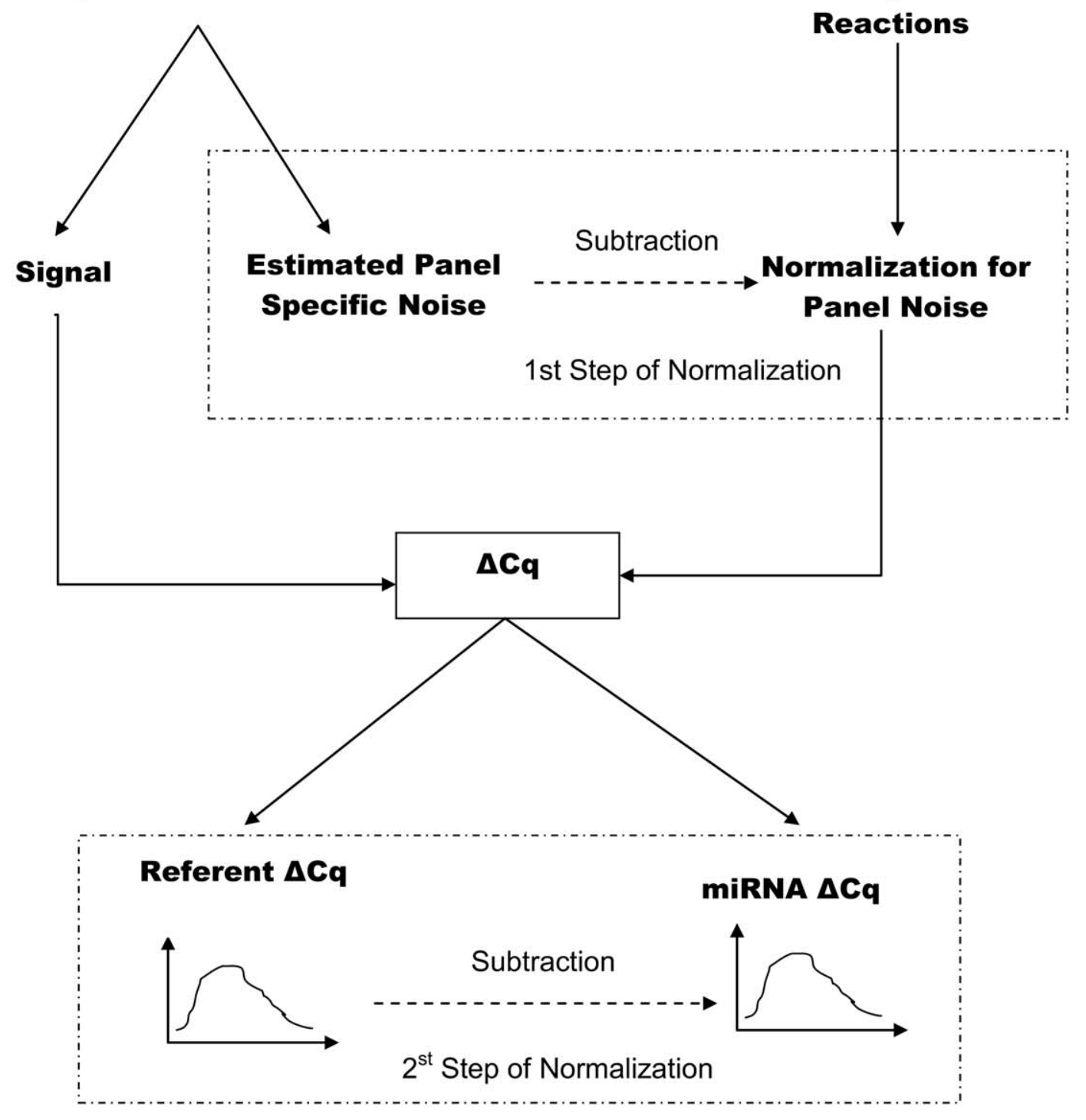

Figure 1. Schematic representation of the normalization procedure and estimation of relative fold changes adopted in the manuscript. Replicate qPCR reactions were analyzed with a hierarchical linear mixed model in order to estimate panel specific correction factors that were subtracted from the raw $C_{q}$ signals of unreplicated reactions (first step), while simultaneously estimating the difference $\left(\Delta C_{q}\right)$ between an experimental and referent state. In the second step, the $\Delta C_{q}$ of the spiked in control was subtracted from the non-control $\Delta C_{q}$ values to calibrate the relative fold changes according to the Delta-Delta method. Both steps of the normalization procedure acknowledged the uncertainty implicit in estimating the $\Delta C_{q}$ of both control and non-control signals (shown as a density plot at the bottom part of the figure), by performing this subtraction probabilistically i.e. by Monte Carlo methods. doi:10.1371/journal.pone.0054662.g001

scatterplots, in which each principal component is plotted against all others. PPCA calculations were performed with the "pcaMethods" package [12] in bioconductor [13].
To analyze the difference in miRNA expression within patient groups, we quantified the relative expression level of each miRNA, its normalized threshold cycle difference $\left(\Delta \mathrm{C}_{\mathrm{q}}\right)$ i.e. the difference 
Table 1. Patient Demographics.

\begin{tabular}{|c|c|c|c|c|}
\hline & \multicolumn{2}{|l|}{ Group A } & \multicolumn{2}{|l|}{ Group B } \\
\hline & \multicolumn{2}{|c|}{ Clinical Classification } & \multicolumn{2}{|l|}{ Clinical Classification } \\
\hline & Normal & Overt Nephropathy & Intermittent Microalbuminuria & Persistent Microalbuminuria \\
\hline $\mathrm{N}$ of subjects & 10 & 10 & 10 & 10 \\
\hline Samples (collected) & 10 & 10 & 20 & 20 \\
\hline Samples(profiled) & 10 & 10 & 19 & 14 \\
\hline Age (yrs) & $42.8 \pm 5.1$ & $41.4 \pm 6$ & $29.4 \pm 6.3$ & $27.5 \pm 5.3$ \\
\hline Women & 5 & 5 & 5 & 5 \\
\hline Duration of Diabetes (yrs) & $34.1 \pm 5.8$ & $34.4 \pm 6.4$ & $20.7 \pm 5.4$ & $21.3 \pm 5.8$ \\
\hline CAD (including MI) & 3 & 1 & 0 & 1 \\
\hline Stroke & 0 & 0 & 0 & 0 \\
\hline PVD & 2 & 2 & 0 & 1 \\
\hline Peripheral Neuropathy & 5 & 5 & 0 & 0 \\
\hline Proliferative Retinopathy & 4 & 1 & 1 & 5 \\
\hline Hypertension & 1 & 6 & 0 & 0 \\
\hline HgBA1c (\%) & $8.2 \pm 1.1$ & $8.2 \pm 1.0$ & $9.9 \pm 1.9$ & $10.2 \pm 2.4$ \\
\hline LDL-c (mg/dl) & $103.5 \pm 20.2$ & $106.3 \pm 44.2$ & $100.3 \pm 20.6$ & $115.9 \pm 57.5$ \\
\hline ACEi or ARB therapy & 1 & 8 & 0 & 0 \\
\hline LDL-c lowering therapy & 1 & 5 & 0 & 0 \\
\hline
\end{tabular}

Abbreviations: CAD (Coronary Artery Disease), MI (Myocardial Infarction), PVD (peripheral vascular disease), HgbA1c (Glycosylated Hemoglobin A1c), LDL-c (Low Density Lipoprotein cholesterol), ACEi (Angiotensin Converting Enzyme Inhibitor), ARB (Angiotensin II Receptor Blocker).

doi:10.1371/journal.pone.0054662.t001

between the quantification cycle in the experimental $(\mathrm{E})$ and the reference $(\mathrm{R})$ state : $\Delta \mathrm{C}_{\mathrm{q}}=\mathrm{C}_{\mathrm{q}}(\mathrm{E})-\mathrm{C}_{\mathrm{q}}(\mathrm{R})$, with positive $\Delta \mathrm{C}_{\mathrm{q}}$ values indicating lower concentrations. To ensure a sufficient amount of data for downstream analyses, only those miRNAs that were detected in at least 2/3 of patient samples in each comparison were analyzed.

A mixed effects model was used simultaneously accounting for matching patients within pairs while normalizing $\Delta \mathrm{C}_{\mathrm{q}}$ values for PCR related factors. Normalization of quantification cycle signals occurred in two steps (Figure 1): first, we developed a regression model that utilized the multiple replicates in the qPCR panels to decompose the corresponding measurements into signal and (panel/PCR) specific noise factors. Secondly, the difference in the expression level of the spiked in (UniSp3) control was used to calibrate relative fold changes $(F C)$ by the Delta-Delta method $[14,15]$ as: $\mathrm{FC}=2^{-\Delta \Delta \mathrm{Cq}}$, where $\Delta \Delta \mathrm{C}_{\mathrm{q}}=\Delta \mathrm{C}_{\mathrm{q}}($ miRNA $)-\Delta \mathrm{C}_{\mathrm{q}}(\mathrm{UniSp} 3)$. The parameters of the regression model were estimated from a Bayesian probabilistic viewpoint, a decision justified by the exploratory, hypothesis generating [16] nature of this work and the amenability of the complex mixed models utilized to Bayesian computational methods. In this study we used "objective", likelihood-dominated, non-informative priors [17] due to the lack of previous information that could be used to specify prior beliefs for the levels of the miRNAs examined.

Bayesian models were programmed in the BUGS language (Text S1 sections Bayesian Computations and WinBUGS code) [18]. Statistics (means, standard errors) of posterior probability MCMC samples were used to summarize inferences about individual $\Delta \mathrm{C}_{\mathrm{q}}$ values, while the degree to which each estimated $\Delta \mathrm{C}_{\mathrm{q}}$ differs from zero was quantitated by means of symmetric pseudocontour probabilities which can be viewed as Bayesian analogs of p-values [19]. 95\% Posterior Density Credible (symmetric)
Intervals $(\mathrm{CrI})$ were used to provide a range of values in which the estimated $\Delta \mathrm{C}_{\mathrm{q}}$ lie with a probability of $95 \%$.

miRNA target functional profiling. To infer putative targets of differentially expressed miRNAs we utilized three different algorithms: miRanda (release August 2010) [20], TargetSCan (release 6, November 2011) [21] and miRDB (version 4.0, January 2012) [22]. In order to declare a specific mRNA as a target of a given miRNA species, we required that at least 2 of the 3 databases predict the latter to bind to the former. To leverage the quantitative urinary expression profiles and miRNA target database information into more concrete predictions we appealed to a biochemical argument based on Hill plots. In this approximation for the interaction between miRNA and mRNA, the fraction of the bound sites $(\theta)$ is related to the free ligand concentration $(L)$ and the dissociation constant $\left(K_{d}\right)$ by the logistic equation:

A change in the ligand concentration between an experimental state $\left(L_{E}\right)$ and the reference $\left(L_{R}\right)$ is related to a change in the fraction of bound sites which can be expressed in terms of the relative fold change. Hence, by the above expression:

To the extent that miRNAs function as negative regulators of mRNA translation a positive log-odds ratio (larger bound fraction) would imply a propensity for the target mRNA expression to be reduced in the experimental state.

To synthesize the evidence from multiple $\Delta \Delta C_{t}$ values of miRNAs targeting a specific gene we used the means and standard errors from the MCMC simulations as input to random effects metaanalyses. Such techniques, allow one to test the hypotheses that a given sample of $\Delta \Delta C_{q}$ 's ("treatment effects") follows a distribution with a mean that departs from zero. In such a case, one would expect the mRNA profile to deviate to a direction opposite to the 


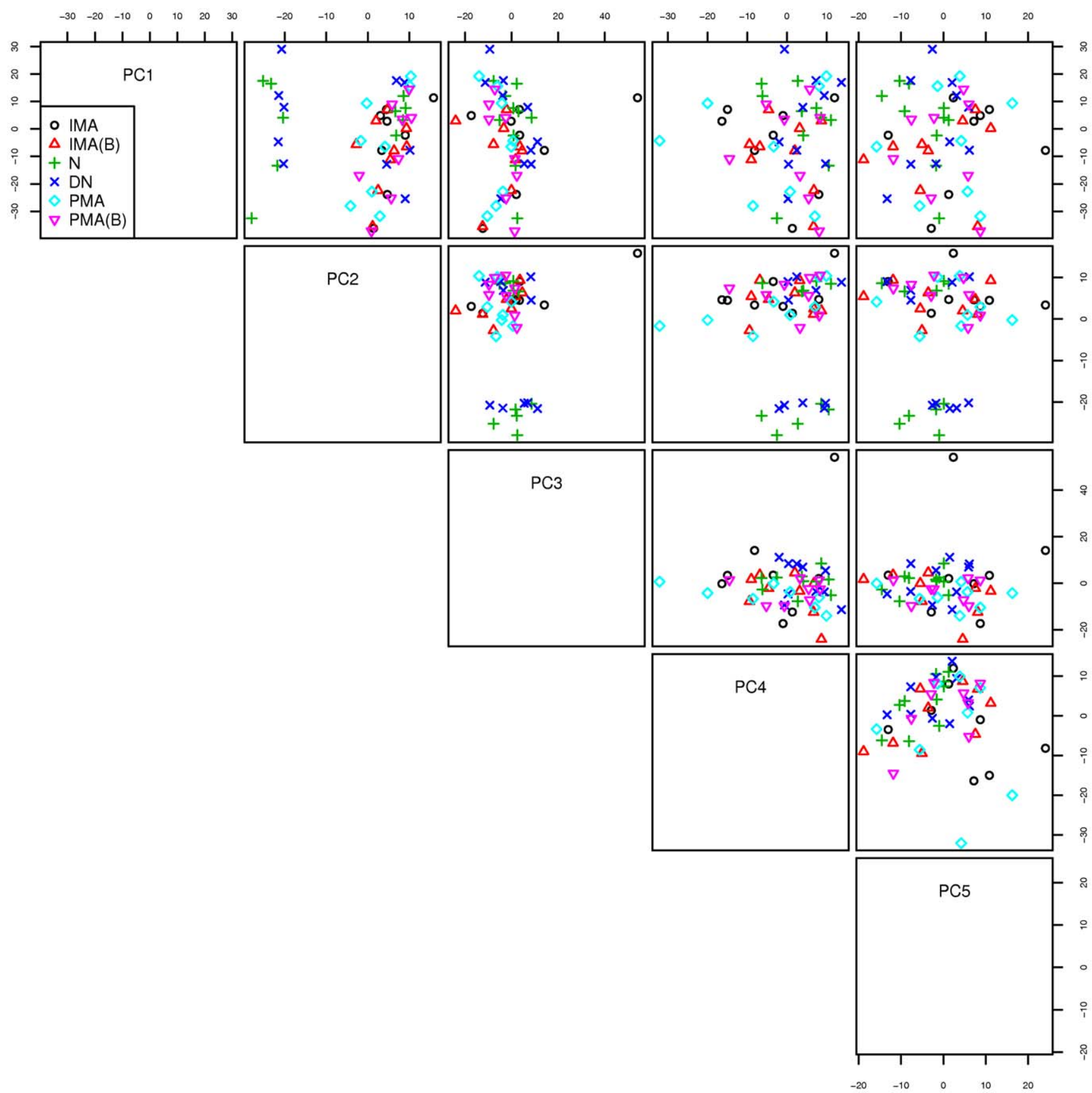

Figure 2. Results of Principal Component Analysis applied to all urine samples analyzed in this study. To present the results of the five dimensional PCA, we utilized bivariate projections in which each component is plotted against all e.g. the second plot in the first row plots the first principal component (PC1) against the second (PC2). Each individual urine sample is color and symbol coded according to the disease classification at the time it was collected. $\mathrm{N}$ : patients without nephropathy, DN: patients with overt nephropathy, IMA(B): normoalbuminuric samples from patients who had intermittent microalbuminuria, PMA(B): last normoalbuminuric samples from patients who had persistent albuminuria, IMA: microalbuminuric samples from patients who had intermittent micro-albuminuria, PMA: micro-albuminuric samples from patients who had persistent microalbuminuria.

doi:10.1371/journal.pone.0054662.g002

miRNA distributional mean (under-expressed miRNA implies overexpression of the cognate mRNA and vice versa). Hence, by considering the log-odds ratios for all putative miRNA-mRNA pairs we estimated functional expression profiles for the miRNA targets for post hoc exploration (enriched term analysis) in the REACTOME [23] and Gene Ontology Project [24].

\section{Results}

\section{Patients and Measurements}

Baseline characteristics of patients included in this study are shown in Table 1. In total we studied 40 patients with T1D: 10 who never developed diabetic renal disease $(\mathrm{N})$ matched against 10 patients who went on to develop overt nephropathy (DN), 10 


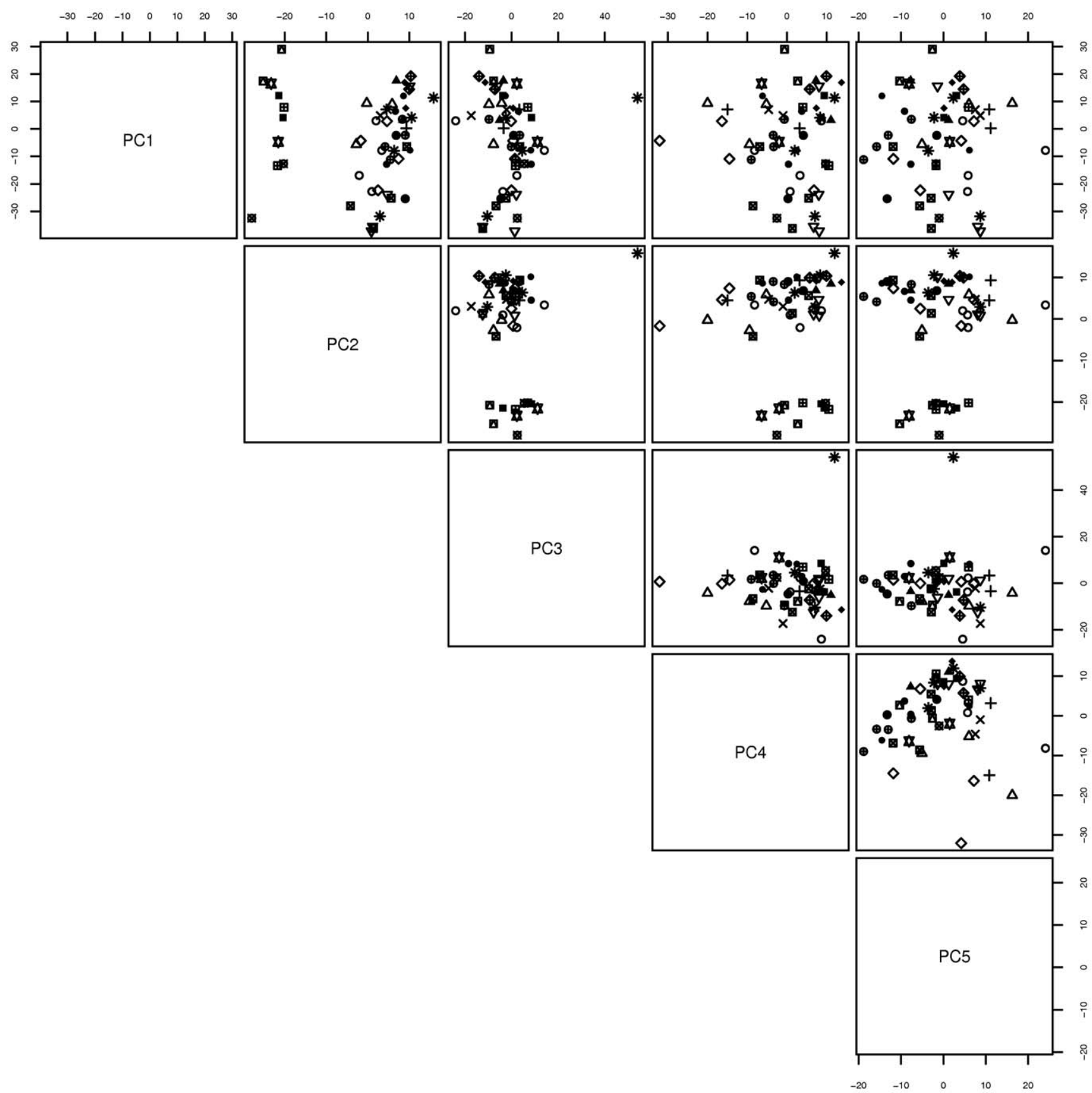

Figure 3. Results of Principal Component Analysis rendered according to pair identification number. This figure utilizes the same bivariate projection setup as Figure 2, but points are symbol coded according to the unique identifier used when matching patients into pairs. For patients with MA who contributed two samples (one at the baseline and one at the microalbuminuric state) there are more than 2 points with the same symbol.

doi:10.1371/journal.pone.0054662.g003

patients with intermittent microalbuminuria (IMA) matched against 10 patients with persistent (PMA) microalbuminuria. In general, patients were well matched within each of the two comparison groups (IMA vs. PMA, $\mathrm{N}$ vs. DN) in terms of their demographics and glycemic control (HBAlc). Roughly $50 \%$ of patients with DN and an equivalent proportion of patients without renal disease had at least one diabetic complication (most commonly peripheral neuropathy). On the other hand, patients at the microalbuminuria group were free of diabetic complications at the time of urine collection. The majority of the patients in this cohort were not on inhibitors of the angiotensin system (i.e. Angiotensin Enzyme Inhibitors or Angiotensin Receptor Blockers), with the exception of patients with overt nephropathy who were receiving them (8/10 patients). Furthermore, these patients were more likely to receive additional agents for blood pressure control, paralleling the severity of their renal disease.

Due to the insufficient amount of RNA, we did not obtain good quality mRNA measurement in 6 samples from 3 patient pairs in the PMA sub-group and one urine sample from the IMA group. Nevertheless, reproducibility of un-normalized $\mathrm{C}_{\mathrm{q}}$ signals from 
Table 2. Differentially expressed miRNAs between albuminuric and non-albuminuric (reference) samples from patients with MA.

\begin{tabular}{|c|c|c|c|}
\hline \multirow[t]{2}{*}{ miRNA } & Fold Change & 95\% Credible Interval & $\mathbf{P}$ \\
\hline & \multicolumn{3}{|c|}{ Under-expressed } \\
\hline $\begin{array}{l}\text { hsa-miR-323b-5p } \\
\text { hsa-miR-453 }\end{array}$ & 0.07 & $0.01-0.42$ & 0.0030 \\
\hline $\begin{array}{l}\text { hsa-miR-221-3p } \\
\text { hsa-miR-221 }\end{array}$ & 0.15 & $0.03-0.80$ & 0.0280 \\
\hline hsa-miR-524-5p & 0.19 & $0.04-0.88$ & 0.0350 \\
\hline \multirow[t]{2}{*}{ hsa-miR-188-3p } & 0.28 & $0.08-0.98$ & 0.0454 \\
\hline & \multicolumn{3}{|c|}{ Over-expressed } \\
\hline $\begin{array}{l}\text { hsa-miR-214-3p } \\
\text { hsa-miR-214 }\end{array}$ & 8.71 & $1.97-38.05$ & 0.0050 \\
\hline $\begin{array}{l}\text { hsa-miR-92b-5p } \\
\text { hsa-miR-92b * }\end{array}$ & 8.65 & $1.11-67.46$ & 0.0394 \\
\hline hsa-miR-765 & 7.22 & $1.78-30.98$ & 0.0046 \\
\hline hsa-miR-429 & 5.92 & $1.42-23.94$ & 0.0136 \\
\hline $\begin{array}{l}\text { hsa-miR-373-5p } \\
\text { hsa-miR-373* }\end{array}$ & 4.50 & $1.19-17.27$ & 0.0296 \\
\hline hsa-miR-1913 & 4.37 & $1.30-15.47$ & 0.0156 \\
\hline hsa-miR-638 & 3.71 & $1.02-13.81$ & 0.0464 \\
\hline
\end{tabular}

For miRNAs whose name changed after the introduction of the $18^{\text {th }}$ version of MiRBase, we provide both the previous (in italics) and the recent (regular font) name.

doi:10.1371/journal.pone.0054662.t002

urine in the rest of the samples was high (Dataset S1 for unnormalized $\mathrm{C}_{\mathrm{q}}$ values). The quality of miRNA measurements was assessed by using the detection probability of controls (Fig. S1) and the reproducibility of un-normalized signals in replicates present in the qPCR panels (Fig. S2). The detection probability of negative (BLANK) and positive (UniSp6 and UniSp3) controls matched the expected ratios $(0 \%, 50 \%$ and $100 \%$ respectively). The corresponding $\mathrm{C}_{\mathrm{q}}$ values for the spiked controls were much less variable than those of endogenous (miRNAs and small RNA) controls suggested by the platform manufacturer without evidence of substantial inter-plate variability. Hence fold changes were computed relative to the UniSp3 RNA for all subsequent comparisons.

\section{Principal Component Analysis}

A global view of the changes in urinary miRNA profiles according to the clinical classification was performed with PCA and the results for the first five principal components $(\mathrm{PC})$ are shown in Figure 2. Samples from Group A (DN vs. N) were grouped together in some projections (e.g. see second plot in the first row depicting PC1 v.s. PC2), while in other projections (PC3 vs. PC4 show in the last plot from the left, second row) samples from both comparisons grouped together. Although patients with DN appear to form a cluster distinct from those who never developed nephropathy (projections PC2 v.s.PC3-5, in the second row) there was no obvious clustering structure in the profiles of patients with MA at either the baseline or the microalbuminuric state).

To explore whether patients who had been matched into pairs had similar microRNA profiles we plotted the results of the PCA according to the pair identifier. These results which are shown in
Figure 3 show that patients within pairs have similar profiles (the distance between points with the same plotting symbol appears smaller than the distance of points from patients from different pairs). Taken together these data suggest that there global changes in the miRNA profile associated with the different stages of diabetic nephropathy. Furthermore, the clustering of profiles with patient pairs suggests that there are other determinants of microRNA expression which correlate with the variables we used to match patients during sample selection.

\section{Comparisons between IMA and PMA}

219 miRNAs yielded measurable signals in $>75 \%$ of the 33 urine samples profiled for this comparison. We observed only a few differences in the baseline samples. Relative to the IMA group, patients with PMA demonstrate decreased miR-323b-5p levels (Fold Change (FG) 0.13, 95\% CrI: 0.03-0.67, p=0.014) and increased levels of: $m i R-122-5 p$ (FC: $7.45,95 \%$ CrI: $1.77-32.05$, $\mathrm{p}=0.006), \operatorname{miR}-429$ (FC: 4.72, 95\% CrI: $1.14-19.86, \mathrm{p}=0.034)$, at baseline.

Table 2 shows the miRNAs with altered levels in the MA samples relative to baseline. Appearance of micro-albuminuria is associated with decreased levels of $m i R-323 b-5 p$ and increased urine concentration of miR-429. In Table 3 we summarize the incremental FCs of specific miRNA levels in microalbuminuric samples between PMA and IMA patients. Of note, two miRNAs in the PMA patients (miR-373-5p and miR-323b-bp) exhibit concentration changes that are in the opposite direction relative to the changes observed when both IMA/PMA patients manifest MA. There were no further changes observed in the levels of the remaining miRNAs found to be different in Table 2, while only a small number of miRNAs appear to show incremental concentration changes in the microalbuminuric urine from PMA patients.

Table 3. Incremental differential expression of miRNAs between albuminuric samples from patients with persistent microalbuminuria (PMA) relative to patients with intermittent microalbuminuria (IMA).

\begin{tabular}{|c|c|c|c|}
\hline \multirow{2}{*}{ miRNA } & $\begin{array}{l}\text { Fold } \\
\text { Change }\end{array}$ & 95\% Credible Interval & $\mathbf{P}$ \\
\hline & \multicolumn{3}{|c|}{ Under-expressed } \\
\hline $\begin{array}{l}\text { hsa-miR-589-5p } \\
\text { hsa-miR-589 }\end{array}$ & 0.05 & $0.00-0.98$ & 0.048 \\
\hline $\begin{array}{l}\text { hsa-miR-373-5p } \\
\text { hsa-miR-373* }\end{array}$ & 0.07 & $0.01-0.45$ & 0.007 \\
\hline hsa-mir-520h & 0.12 & $0.02-0.80$ & 0.026 \\
\hline \multirow{2}{*}{$\begin{array}{l}\text { hsa-miR-92a-3p } \\
\text { hsa-miR-92a }\end{array}$} & 0.14 & $0.02-0.98$ & 0.048 \\
\hline & \multicolumn{3}{|c|}{ Over-expressed } \\
\hline $\begin{array}{l}\text { hsa-miR-323b-5p } \\
\text { hsa-miR-453 }\end{array}$ & 31.51 & $2.91-368.48$ & 0.0044 \\
\hline hsa-miR-433 & 16.24 & $1.39-196.0$ & 0.028 \\
\hline $\begin{array}{l}\text { hsa-miR-17-5p } \\
\text { hsa-miR-17 }\end{array}$ & 14.82 & $1.09-214.0$ & 0.044 \\
\hline $\begin{array}{l}\text { hsa-miR-222-3p } \\
\text { hsa-miR-222 }\end{array}$ & 11.22 & $1.13-102.0$ & 0.036 \\
\hline hsa-let-628-5p & 7.59 & $1.07-52.2$ & 0.044 \\
\hline
\end{tabular}


Table 4. Differentially expressed miRNA between patients who developed overt diabetic nephropathy relative to patients who did not.

\begin{tabular}{|c|c|c|c|}
\hline \multirow{2}{*}{ miRNA } & Fold Change & 95\% Credible Interval & $\mathbf{P}$ \\
\hline & \multicolumn{3}{|c|}{ Under-expressed } \\
\hline \multirow{2}{*}{$\begin{array}{l}\text { hsa-miR-221-3p } \\
\text { hsa-miR-221 }\end{array}$} & 0.25 & $0.07-0.86$ & 0.0330 \\
\hline & \multicolumn{3}{|c|}{ Over-expressed } \\
\hline hsa-miR-619 & 6.98 & $1.86-27.80$ & 0.0030 \\
\hline hsa-miR-486-3p & 6.43 & $1.36-26.66$ & 0.0290 \\
\hline $\begin{array}{l}\text { hsa-miR-335-5p } \\
\text { hsa-miR-335 }\end{array}$ & 5.81 & $1.70-20.71$ & 0.0050 \\
\hline hsa-miR-552 & 5.47 & $1.19-27.50$ & 0.0310 \\
\hline hsa-miR-1912 & 4.72 & $1.01-23.92$ & 0.0490 \\
\hline hsa-miR-1224-3p & 4.45 & $1.10-17.48$ & 0.0430 \\
\hline $\begin{array}{l}\text { hsa-miR-424-5p } \\
\text { hsa-miR-424 }\end{array}$ & 4.38 & $1.35-15.13$ & 0.0130 \\
\hline $\begin{array}{l}\text { hsa-miR-141-3p } \\
\text { hsa-miR-141* }\end{array}$ & 3.81 & $1.29-11.17$ & 0.0140 \\
\hline $\begin{array}{l}\text { hsa-miR-29b-1-5p } \\
\text { hsa-miR-29b-1* }\end{array}$ & 3.03 & $1.09-8.61$ & 0.0370 \\
\hline
\end{tabular}

For miRNAs whose name changed after the introduction of the 18th version of MiRBase, we provide both the previous (in italics) and the recent (regular font) name.

doi:10.1371/journal.pone.0054662.t004
Of note miR-324-3p, a demonstrated a trend towards a higher incremental change in expression level (FG 2.90, 95\% CrI: 0.53$17.62, \mathrm{p}=0.11)$ in patients with PMA.

\section{Comparisons between Patients with and without DN}

283 miRNAs yielded measurable signals in $>75 \%$ of the 20 urine samples from these 10 patient pairs. In Table 4 we summarize the miRNAs with altered expression in the urine of patients with nephropathy. With the exception of miRNA-221-3p, which decreased similar to the comparison of follow up and baseline MA samples, the remaining miRNAs did not demonstrate altered expression in any of the previous comparisons. Finally, there was a trend for miR-589 and miR-323b-5p to be increased in the urine of patients with overt nephropathy. The corresponding FCs were 2.99, (95\% CrI: 0.81-9.95, $\mathrm{p}=0.087)$ and 4.45, (95\% CrI: 090-29.1, $\mathrm{p}=0.08$ ).

\section{miRNA Target Functional Profiling}

Figure 4 summarizes the number of predicted mRNA targets of the differentially expressed miRNAs in diabetic urine based on the prediction databases. Analysis of enriched terms in REACTOME (Table 5) suggest that the predicted miRNA targets map to a distinct pathways involving growth factor signaling, apoptosis, immunity, substrate metabolism, transmembrane transport and certain non-kidney related terms. Furthermore, the identified pathways overlapped considerably between the comparisons of patients with overt nephropathy and normals, and follow-up v.s. baseline samples from MA patients. In the comparisons within baseline and follow-up MA samples we found only a few $(<80)$ targets mapping to annotated REACTOME pathways, thus

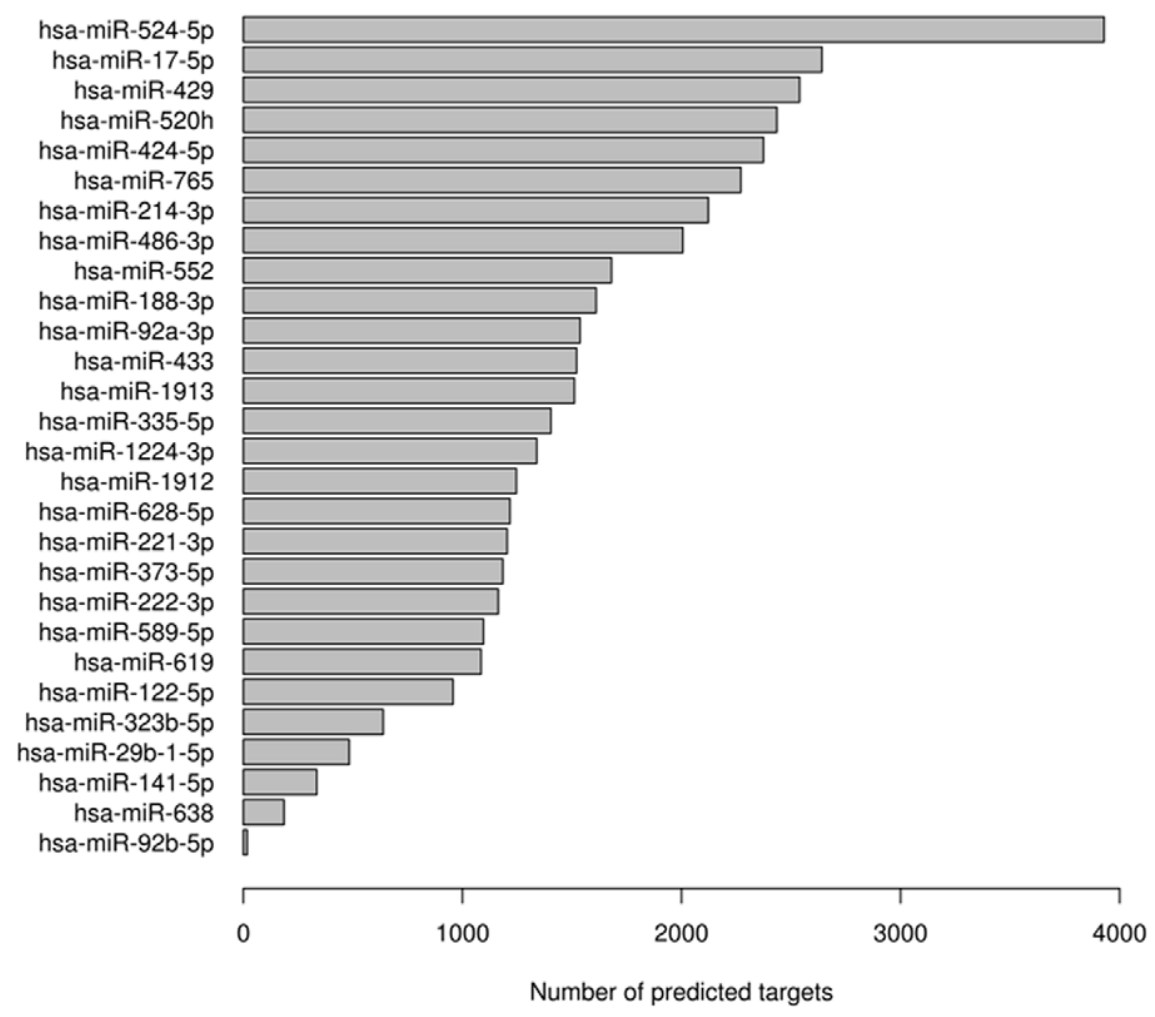

Figure 4. Distribution of the number of mRNAs targeted by differentially regulated microRNAs in diabetic urine. doi:10.1371/journal.pone.0054662.g004 
Table 5. REACTOME pathway terms enriched in targets of differentially expressed miRNAs.

\begin{tabular}{|c|c|c|c|c|}
\hline \multirow[b]{2}{*}{ Pathway } & \multicolumn{2}{|c|}{$\begin{array}{l}\text { Albuminuric vs Normoalbuminuric } \\
\text { in the MA group }\end{array}$} & \multicolumn{2}{|c|}{ Overt vs Normal } \\
\hline & P-value & Fraction & P-value & Fraction \\
\hline \multicolumn{5}{|l|}{ Signal Transduction } \\
\hline Signaling by SCF-KIT & 0.006 & $18 / 76$ & 0.001 & $41 / 76$ \\
\hline Signaling by Insulin receptor & 0.009 & 23/109 & $<0.001$ & $65 / 109$ \\
\hline Signaling by NGF & 0.016 & $38 / 212$ & $<0.001$ & $119 / 212$ \\
\hline Signaling by Rho GTPases & 0.024 & $24 / 125$ & $<0.001$ & $71 / 125$ \\
\hline Signaling by ERBB4 & 0.027 & $16 / 76$ & $<0.001$ & $45 / 76$ \\
\hline Signaling by ERBB2 & 0.035 & 19/97 & $<0.001$ & $59 / 97$ \\
\hline Signaling by PDGF & 0.040 & $22 / 118$ & $<0.001$ & $67 / 118$ \\
\hline Signaling by VEGF & 0.041 & $4 / 11$ & & \\
\hline Signaling by EGFR & 0.044 & 20/106 & $<0.001$ & $64 / 106$ \\
\hline Dowstream signaling of activated FGFR & 0.038 & 19/98 & $<0.001$ & $61 / 98$ \\
\hline Signaling by BMP & & & 0.001 & $16 / 23$ \\
\hline Signaling by TGF $\beta$ & & & 0.004 & $11 / 15$ \\
\hline DAG and IP3 signaling & & & 0.010 & $20 / 31$ \\
\hline PIP3 activates AKT signaling & & & 0.020 & $15 / 26$ \\
\hline RAF/MAP kinase cascade & & & 0.031 & $7 / 10$ \\
\hline Signaling by Notch & & & 0.036 & $13 / 23$ \\
\hline Interaction of integrin $\alpha 5 \beta 3$ with fibrillin & 0.044 & $2 / 3$ & & \\
\hline Interaction of integrin $\alpha 5 \beta 3$ with von Willbrand factor & 0.044 & $2 / 3$ & & \\
\hline Integrin cell surface interactions & & & 0.024 & $40 / 85$ \\
\hline Cell-Cell Communication & & & 0.009 & $57 / 122$ \\
\hline \multicolumn{5}{|l|}{ Cell Cycle } \\
\hline G0 and early G1 & & & 0.040 & $12 / 21$ \\
\hline \multicolumn{5}{|l|}{ Metabolism } \\
\hline Metabolism of lipids and lipoproteins & 0.022 & $51 / 305$ & 0.005 & $132 / 205$ \\
\hline Cysteine formation from homocysteine & 0.016 & $2 / 2$ & & \\
\hline Integration of energy metabolism & & & 0.009 & $45 / 93$ \\
\hline \multicolumn{5}{|l|}{ Metabolism of proteins } \\
\hline Post-translational protein modification & 0.045 & $30 / 173$ & 0.019 & $76 / 173$ \\
\hline Transmembrane transport of small molecules & 0.007 & $67 / 396$ & $<0.001$ & 189/396 \\
\hline Membrane trafficking & & & 0.032 & $40 / 84$ \\
\hline \multicolumn{5}{|l|}{ Apoptosis } \\
\hline Caspase- 8 is formed from procaspase- 8 & 0.019 & $4 / 9$ & & \\
\hline \multicolumn{5}{|l|}{ Gene Expression } \\
\hline RNA Polymerase II Transcription & 0.050 & 19/101 & & \\
\hline Capping complex formation & 0.039 & $7 / 26$ & & \\
\hline Nuclear Receptor Transcription & & & 0.005 & $28 / 51$ \\
\hline \multicolumn{5}{|l|}{ Steroid hormones } \\
\hline Vitamin D (calciferol) metabolism & 0.048 & $3 / 7$ & & \\
\hline Activated AMPK stimulates fatty-acid oxidation in muscle & & & 0.008 & $12 / 18$ \\
\hline \multicolumn{5}{|l|}{ Neuronal System } \\
\hline Heterodimerization of CEACAMs & & & 0.047 & $3 / 3$ \\
\hline Transmission across Chemical Synapses & & & $<0.001$ & $66 / 108$ \\
\hline \multicolumn{5}{|l|}{ Immune System } \\
\hline Interleukin-2 signaling & 0.029 & $10 / 41$ & & \\
\hline 14-3-3 zeta binding allows recruitment of PI3K & 0.033 & $5 / 15$ & & \\
\hline Signaling by interleukins & & & 0.002 & $54 / 105$ \\
\hline
\end{tabular}


Table 5. Cont.

\begin{tabular}{|c|c|c|c|c|}
\hline \multirow[b]{2}{*}{ Pathway } & \multicolumn{2}{|c|}{$\begin{array}{l}\text { Albuminuric vs Normoalbuminuric } \\
\text { in the MA group }\end{array}$} & \multicolumn{2}{|c|}{ Overt vs Normal } \\
\hline & P-value & Fraction & P-value & Fraction \\
\hline Hemostasis & & & $<0.001$ & $206 / 426$ \\
\hline Platelet homeostasis & 0.008 & $14 / 56$ & & \\
\hline Platelet activation, signaling and aggregation & 0.011 & $35 / 187$ & & \\
\hline
\end{tabular}

precluding a meaningful assessment with this structured vocabulary. The results from Gene Ontology (GO) analysis (Datasets S2, S3, S4, S5) were consistent with REACTOME, and also identified enrichment of terms relating to nitrogen compound metabolism, Golgi/membrane/ER vesicle recycling, ubiquitin-dependent degradation, cell adhesion and cell adhesion. In addition, GO analyses also suggested the enrichment of renal (GOBPID: 0072166, $\mathrm{p}=0.03$ ) and non-renal developmental (GOBPID:0048557, $\mathrm{p}=0.0002$, GOBPID:0060174, $\mathrm{p}=0.002$ ) pathways, myoblast determination (GOBPID:007518, $\mathrm{p}=0.0002$ ) innate immunity (GOBPID:0002717, $\mathrm{p}=0.001$ ) and free radical generation/oxidative stress (GOBPID:0071371/0071450-51, $\mathrm{p}=0.01)$.

\section{Discussion}

In this paper we report the changes of urinary miRNA spectrum in T1D patients with different stages of albuminuria and nephropathy. We found concentration changes on specific miRNAs that may involve in specific pathways known to be altered in various forms of renal diseases. Since the kidney is the most likely source of these urinary miRNAs, we suggest that these miRNAs may be of biological and clinical significance in T1D.

A global Principal Component Analysis viewpoint of the microRNA profiles analyzed in this report suggests that there are some differences in the expression of urinary microRNA which appear to follow the clinical classification of patients and urinary samples with respect to albumin excretion. The apparent clustering of profiles from patients who had been matched into pairs, suggests that there are other factors affecting urinary microRNA besides the clinical classification of disease. Such factors are likely related to the variables we used in patient matching e.g. age, sex, and duration of disease and level of glycemic control. This observation justifies post-hoc our decision to explore specific microRNA signatures across the spectrum of clinical classification of patients and samples using a matched case control design.

Our matched case-control Bayesian analyses highlight a set of 27 differentially regulated miRNAs across different clinical stages of diabetic renal disease. Previous work using experimental, clinical chemistry or biopsy samples has demonstrated differential expression of many of these miRNAs in a variety of renal conditions: hypertensive nephrosclerosis (with an increase of miR-429 levels in human renal biopsies [25]), mouse models of chronic renal injury (increased miR-214 levels [26]) and renal senescence [27] (increased miR-335 levels). Other miRNAs have also been implicated in immunologically mediated renal diseases such as lupus nephritis(miR-429 [28], miR-638,miR-373-5p and miR-92b5p [29]), IgA nephropathy (miR-429 correlating with the level of proteinuria and renal function [30]), and acute $T$ cell rejection of renal allografts (decreased miR-323-5p, miR-638/miR-373-5p [31]).

Based on miRNA target prediction databases, miRNAs showing concentration changes in diabetic urine may regulate genes that play key roles in renal physiology and pathophysiology: fibronectin a key component of the extracellular matrix that accumulates in diabetic nephropathy [32] (miR-17-5p [33] which is also regulated in senescence models of renal proximal tubule epithelial cells [34]), PKD2 responsible for polycystic kidney disease (miR-17-5p $[35,36])$, Sod2, superoxide dismutase, a mitochondrial antioxidant enzyme in renal mesangial cells(miR-335 [27]), Claudin-16 a key component of the tight junction in the thick ascending limb (hasmiR-323b-5p [37]), the tumor suppressor protein PTEN which is decreased in DN [38] (and is directly regulated by miR-221-3p/ 222-3p [39] in heterologous systems), Abcg2 (a stem cell marker [40] regulated by miR-520h [41]), $\operatorname{Vhl}$ (a tumor suppressor gene involved in renal tumours targeted by miR-92a [42]). Hence, prior research highlights a kidney related role for a number of the miRNAs found to be differentially expressed in our analyses, suggesting that these miRNAs may be important mediators of renal damage rather than simple biomarkers of an underlying injury process without pathobiological significance.

In addition, intriguing connections in heterologous systems have been reported for other miRNAs highlighted in this report: miR221-3p/222-3p (neovascularization and vascular neointimal hyperplasia [43], Advanced Glycosylation End product mediated vascular damage [44]), miR-424 (regulating angiogenesis in the setting of hypoxia by targeting Cul2 [45] as well as Vegfr2 and Fgfr1 [46]). Many of these conditions have been recognized as clinically important vascular complications of diabetes, often presenting simultaneously with the development of nephropathy; hence one may conjecture that the spectrum of urine miRNAs may allow one to stratify the risk of diabetic patients for developing extrarenal complications.

With the samples used in this study, we could not verify the association of miR-192 with DN. Higher miR-192 levels have been previously linked to renal damage in the streptozocin (T1D) and the $\mathrm{db} / \mathrm{db}(\mathrm{T} 2 \mathrm{D})$ mouse nephropathy models [47] through TGF $\beta$-mediated production of miR-192 by mesangial cells. More recent evidence points towards a positive feedback loop for TGF $\beta$ production involving miR-192 and miR-200b/c in mesangial cells [48]. On the other hand, decreased miR-192 was noted in biopsy specimens of patients with advanced diabetic nephropathy, while miR-192 expression was positively correlated with EGFR and negatively correlated with the degree of fibrosis suggesting a protective role for miR-192 [49]. In that report, miR-192 expression was predominantly localized to tubular epithelial cells and TGF exposure was found to decrease both miR-192 and Ecadherin mRNA levels. Hence it appears that miR-192 may be 
regulated differently in different renal cell populations, possibly in a DN stage specific manner. This hypothesis is supported by recent evidence which failed to detect alterations in miR-192 expression in microdissected glomeruli of Munich Wistar Fromter rat model of spontaneous develop diabetic nephropathy [50]. Since the miRNAs in the urine originate from diverse cellular sources in the kidney, the lack of a differential expression of miR192 in this report may reflect the cancelation of two diverging (positive in mesangial, negative in tubular epithelial cells) signals leading to an overall "null" effect.

Most of the identified miRNAs exhibited changes in one disease state rather than showing a quantitative trend of increasing or decreasing expression paralleling the severity of albuminuria. To understand this pattern we examined the predicted targets of these miRNAs and the corresponding pathways using structured vocabularies for biological annotation. Despite the disparate identity of the miRNAs, the mRNAs that are predicted to be targeted by them map to pathways that have been previously shown to be pathophysiologically relevant to DN: TGF (the prototypical "renal-fibrosis" culprit [51]), PDGF (associated with mesangial proliferation and fibrosis [52]) and FGF (clinical predictor of progression in diabetic nephropathy [53]).

Our analyses suggest the involvement of NGF (Nerve Growth Factor, a prototypical Central Nervous System trophic molecule) in diabetic nephropathy. This may lead to a new direction toward the development of TID associated nephropathy since so far the renal expression of NGF has been thought to reflect the level of glycemic control [54]. Nevertheless, NGF has been recently shown to be involved in tissue repair and fibrosis in liver, skin and lung [55], and its involvement in non-diabetic renal disease has been noted in a number of biopsy studies over the last 30 years [56], so that the association of NGF with diabetic nephropathy appears plausible.

Growth Factor as well as other pathways (e.g. cell-cell and cellmatrix) are targeted from the microalbuminuric stage, while the number of targeted genes in these pathways increased at the overt nephropathy stage. Hence an "exposure-response" relation appears at the target (mRNA) rather than the regulator (miRNA) level. This relation stems from the overlapping, combinatorial, binding specificities of miRNAs to their mRNA targets so that the same pathways may be targeted by rather different sets of miRNAs depending on the prevailing cellular context.

An interesting aspect of the targets associated with the miRNAs identified in this study is the lack of an overwhelming association between growth factor transduction pathways and the tempo of MA. Rather, an association with tissue damage, innate immunity, metabolic pathway and developmental program (re)-activation was shown, suggesting that recurrent bouts of metabolic or free oxidative stress may account for the persistency and possibly the progression of MA to overt nephropathy. To the extent that these statistically determined patterns are verified experimentally, further development of miRNA target identification may have potential clinical implications as an early diagnostic test for diabetic renal disease or to select and or monitor response to emerging therapies for diabetic renal disease; e.g. pentoxifylline [57], pirfenidone [58] and bardoxolone [59] which interfere with pathways implicated in our analyses.

The findings of our study should be interpreted in light of a number of limitations. First, we analyzed urine samples from an era in which current therapies for diabetic nephropathy (angiotensin converting enzyme inhibitors and angiotensin receptor blockers) were not widely used early in the disease process. Hence most of the patients with MA were not on ACEi/ARB inhibition even though evidence from randomized trials suggest that these agents delay the appearance of microalbuminuria [60,61]. On the other hand, most patients with overt nephropathy were on such agents with persistence of their macroalbuminuric state. Hence, our findings reflect the natural urinary miRNA phenotype of the early stages of diabetic nephropathy, the failing treatment one in advance disease and are not proposed to be representative of patients undergoing optimal treatment with these agents. Although this would appear to represent a major limitation of this study, the data presented here are rather unique in that they provide information on both untreated patients as well as those failing therapy, allowing some insight into the pathways that underline treatment resistance to the current treatment paradigm. This is exemplified by miR-324-3p which was apparently increased in patients with PMA not receiving an ACEi in accordance with recent animal data suggesting that this miRNA is a promoter of renal fibrosis and is downregulated by ACEi inhibition. At the same time, our patients with overt nephropathy showed no tendency of this miRNA to change relative to controls (FG was 1.06 in this dataset) suggesting that some of the discordance in miRNA profiles may be the result of therapies preferentially affecting certain miRNA species but not others. Since this investigation never intended to delineate treatment induced changes in urine miRNA profiles, future studies should examine both responders and non-responders at different points in time to determine miRNA correlates of therapeutic success and failure. Second, while our experience is no different from previous studies examining urine miRNA profiles in renal transplantation $[31,62]$, systemic lupus [28] and chronic kidney disease [63], many of the urinary miRNA signals in this analysis were of low magnitude requiring a large number of PCR cycles and careful optimization of qPCR conditions [64] to be detected. Third, we inferred the renal origin of urine miRNAs yet the possibility that the latter derive from other sources such as plasma cannot be ruled out. As the approximate molecular weight of miRNAs $(\sim 6.2-7.2 \mathrm{kDa})$ is below the permselectivity threshold of the glomerular filtration barrier $(\sim 60 \mathrm{kDa})$ it is possible that a substantial portion of circulating plasma miRNAs is ultrafiltered in the urine. Nevertheless, a recent study in chronic kidney disease found a dissociation between plasma and urine miRNA spectrum [63] suggesting a substantial non-plasma source for urine miRNA. To resolve these issues, simultaneous profiling of plasma and urine should be undertaken, a task which was not possible in this report due to the unavailability of plasma samples. Fourth, some of the miRNAs identified as differentially regulated have been found to play a role in non-diabetic renal disease, so that the reported associations may lack disease specificity. We tried to overcome this limitation by combining the changes in miRNA concentrations with the simultaneous predictions of miRNA targets. Most of the pathways identified have been linked to the development of diabetic nephropathy among different animal models and clinical studies which suggests the combination of using specific miRNA levels and its interacting mRNA targets as a general approach to enhance interpretability and specificity of miRNA profiles. Furthermore, the use of panels of markers will be much more informative and can potentially distinguish pathologies that produce overlapping sets of markers.

In summary, a set of 27 differentially miRNAs were identified in matched urine samples from T1D patients with different stages of diabetic nephropathy, whose renal outcomes had been ascertained after prolonged follow up. These miRNAs map to pathways of known relevance to the development of diabetic renal disease, strongly suggesting the renal source of the miRNAs. Our results suggest that a number of miRNAs in urine may serve not only as molecular signatures of distinct clinical phenotypes in diabetic 
nephropathy but also as early indicators of alterations in specific biological processes in the kidney which can be of importance in individualizing emergent therapies for diabetic kidney disease. Further studies are needed to extend these observations in the setting of T2D and clarify the potential utility of these miRNAs in early diagnosis, risk stratification for progression and treatment selection or monitoring.

\section{Supporting Information}

Figure S1 Detection probability (\% of PGR reactions which yielded a signal up to a maximum of $38 \mathrm{cycles} / \mathrm{all}$ PCR reactions utilizing the same primer set) of microRNA controls classified according to patient clinical status. hsa-miR-103/191/423-5p: endogenous microRNA controls in the Exiqon platform per manufacturer, U6/SNORD38B/ SNORD49A: small RNA (non- microRNA) endogenous controls, BLANK: Empty PCR wells, UniSP6: Spiked Control (included in $50 \%$ of plates), UniSP3: Spiked Inter-plate Calibrator (included in $100 \%$ of plates).

(TIF)

Figure S2 Raw signals $\left(G_{t}\right)$ of microRNA controls classified according to patient clinical status and plate (A or B) for each of the 53 qPGR panels used in this study. hsa-miR-103/191/423-5p: endogenous microRNA controls in the Exiqon platform per manufacturer, U6/SNORD38B/ SNORD49A: small RNA endogenous controls, BLANK: Empty PCR wells, UniSP6: Spiked Control (included in 50\% of plates), UniSP3: Spiked Inter-Plate Calibrator (included in 100\% of plates). Signal reproducibility appeared to be higher for the spikedin controls than the endogenous ones; furthermore there did not appear to be a substantial inter-plate difference to justify the use of Inter-Plate Calibration.

(PNG)

Table S1 Names and accession numbers of microRNA species analyzed in this study. miRBase names prior to the $18^{\text {th }}$ release are included as well to facilitate comparison with earlier literature. Retired entries in the $18^{\text {th }}$ release of miRBase are

\section{References}

1. Molitch ME, DeFronzo RA, Franz MJ, Keane WF, Mogensen CE, et al. (2004) Nephropathy in diabetes. Diabetes Care 27 Suppl 1: S79-83.

2. Kanwar YS, Sun L, Xie P, Liu F-Y, Chen S (2011) A glimpse of various pathogenetic mechanisms of diabetic nephropathy. Annu Rev Pathol 6: 395423. doi:10.1146/annurev.pathol.4.110807.092150.

3. Carthew RW, Sontheimer EJ (2009) Origins and Mechanisms of miRNAs and siRNAs. Cell 136: 642-655. doi:10.1016/j.cell.2009.01.035.

4. Lee RC, Ambros V (2001) An extensive class of small RNAs in Caenorhabditis elegans. Science 294: 862-864. doi:10.1126/science.1065329.

5. Guo H, Ingolia NT, Weissman JS, Bartel DP (2010) Mammalian microRNAs predominantly act to decrease target mRNA levels. Nature 466: 835-840. doi:10.1038/nature09267.

6. Bartel DP (2004) MicroRNAs: genomics, biogenesis, mechanism, and function. Cell 116: 281-297.

7. Kantharidis P, Wang B, Carew RM, Lan HY (2011) Diabetes complications: the microRNA perspective. Diabetes 60: 1832-1837. doi:10.2337/db11-0082.

8. Bhatt K, Mi Q-S, Dong Z (2011) microRNAs in kidneys: biogenesis, regulation, and pathophysiological roles. Am J Physiol Renal Physiol 300: F602-610. doi:10.1152/ajprenal.00727.2010.

9. Kato M, Arce L, Natarajan R (2009) MicroRNAs and their role in progressive kidney diseases. Clin J Am Soc Nephrol 4: 1255-1266. doi:10.2215/ CJN.00520109.

10. Lorenzen JM, Haller H, Thum T (2011) MicroRNAs as mediators and therapeutic targets in chronic kidney disease. Nat Rev Nephrol 7: 286-294. doi:10.1038/nrneph.2011.26.

11. Roweis, Sam T. (1998) EM Algororithms for PCA and SPCA. Advances in Neural Information Processing Systems. Colorado, USA, 1997: The MIT Press, Vol. 10. 626-632. marked as "DEAD"; in the case of microRNA species not present in a particular database a "NA" entry was included in the table. (XLS)

Text S1 Supplementary Methods and Bayesian Software Code.

(PDF)

Dataset $S 1$ Un-normalized $G_{q}$ values from individual patient experiments.

(ZIP)

Dataset S2 GO term enrichment analysis of targets of differentially expressed microRNAs in baseline (normoalbuminuric) urine samples from patients with PMA versus patients with IMA.

(ZIP)

Dataset S3 GO term enrichment analysis of targets of differentially expressed microRNAs in follow up (microalbuminuric) urine samples from patients with PMA versus patients with IMA.

(ZIP)

Dataset S4 GO term enrichment analysis in follow-up versus baseline samples from PMA patients.

(ZIP)

Dataset S5 GO term enrichment analysis of targets of differentially expressed microRNAs in urine samples from patients with overt nephropathy versus patients without nephropathy.

(ZIP)

\section{Acknowledgments}

We thank Ms. Yue Yuan for RNA isolation.

\section{Author Contributions}

Conceived and designed the experiments: JJ DG TO KW CA JB DE. Performed the experiments: KW SM DH. Analyzed the data: CA KW. Contributed reagents/materials/analysis tools: KW DG TO DE SM DH CA. Wrote the paper: CA JJ KW DG JB TO DE.

12. Stacklies W, Redestig H, Scholz M, Walther D, Selbig J (2007) pcaMethods-a bioconductor package providing PCA methods for incomplete data. Bioinformatics 23: 1164-1167. doi:10.1093/bioinformatics/btm069.

13. Gentleman RC, Carey VJ, Bates DM, Bolstad B, Dettling M, et al. (2004) Bioconductor: open software development for computational biology and bioinformatics. Genome Biol 5: R80. doi:10.1186/gb-2004-5-10-r80.

14. Pfaffl MW (2001) A new mathematical model for relative quantification in realtime RT-PCR. Nucleic Acids Res 29: e45.

15. Schmittgen TD, Lee EJ, Jiang J, Sarkar A, Yang L, et al. (2008) Real-time PCR quantification of precursor and mature microRNA. Methods 44: 31-38. doi:10.1016/j.ymeth.2007.09.006.

16. Box GEP, Tiao GC (1973) Nature Of Bayesian Inference. Bayesian Inference In Statistical Analysis. Wiley Classics Library. 1-75.

17. The Case for Objective Bayesian Analysis (2006). Bayesian Analysis 1: 385-402. doi:10.1214/06-BA115.

18. Lunn D, Spiegelhalter D, Thomas A, Best N (2009) The BUGS project: Evolution, critique and future directions. Stat Med 28: 3049-3067. doi:10.1002/ $\operatorname{sim} .3680$.

19. Held L (2004) Simultaneous Posterior Probability Statements from Monte Carlo Output. Journal of Computational and Graphical Statistics 13: 20-35.

20. John B, Enright AJ, Aravin A, Tuschl T, Sander C, et al. (2004) Human MicroRNA Targets. PLoS Biol 2: e363. doi:10.1371/journal.pbio.0020363.

21. Friedman RC, Farh KK-H, Burge CB, Bartel DP (2009) Most mammalian mRNAs are conserved targets of microRNAs. Genome Res 19: 92-105. doi: $10.1101 /$ gr.082701.108.

22. Wang X, El Naqa IM (2008) Prediction of both conserved and nonconserved microRNA targets in animals. Bioinformatics 24: 325-332. doi:10.1093/ bioinformatics/btm595. 
23. Matthews L, Gopinath G, Gillespie M, Caudy M, Croft D, et al. (2009) Reactome knowledgebase of human biological pathways and processes. Nucleic Acids Research 37: D619-D622. doi:10.1093/nar/gkn863.

24. Ashburner M, Ball CA, Blake JA, Botstein D, Butler H, et al. (2000) Gene ontology: tool for the unification of biology. The Gene Ontology Consortium. Nat Genet 25: 25-29. doi:10.1038/75556.

25. Wang G, Kwan BC-H, Lai FM-M, Choi PC-L, Chow K-M, et al. (2010) Intrarenal expression of miRNAs in patients with hypertensive nephrosclerosis. Am J Hypertens 23: 78-84. doi:10.1038/ajh.2009.208.

26. Denby L, Ramdas V, McBride MW, Wang J, Robinson H, et al. (2011) miR-21 and miR-214 are consistently modulated during renal injury in rodent models. Am J Pathol 179: 661-672. doi:10.1016/j.ajpath.2011.04.021.

27. Bai X-Y, Ma Y, Ding R, Fu B, Shi S, et al. (2011) miR-335 and miR-34a Promote renal senescence by suppressing mitochondrial antioxidative enzymes. J Am Soc Nephrol 22: 1252-1261. doi:10.1681/ASN.2010040367.

28. Wang G, Tam LS, Li EKM, Kwan BCH, Chow KM, et al. (2011) Serum and urinary free microRNA level in patients with systemic lupus erythematosus. Lupus 20: 493-500. doi:10.1177/0961203310389841.

29. Dai Y, Sui W, Lan H, Yan Q Huang H, et al. (2009) Comprehensive analysis of microRNA expression patterns in renal biopsies of lupus nephritis patients. Rheumatol Int 29: 749-754. doi:10.1007/s00296-008-0758-6.

30. Wang G, Kwan BC-H, Lai FM-M, Chow K-M, Kam-Tao Li P, et al. (2010) Expression of microRNAs in the urinary sediment of patients with IgA nephropathy. Dis Markers 28: 79-86. doi:10.3233/DMA-2010-0687.

31. Lorenzen JM, Volkmann I, Fiedler J, Schmidt M, Scheffner I, et al. (2011) Urinary miR-210 as a Mediator of Acute T-Cell Mediated Rejection in Renal Allograft Recipients. American Journal of Transplantation: Official Journal of the American Society of Transplantation and the American Society of Transplant Surgeons. Available:http://www.ncbi.nlm.nih.gov/pubmed/ 21812927. Accessed 25 September 2011.

32. Phillips AO, Steadman R, Morrisey K, Martin J, Eynstone L, et al. (1997) Exposure of human renal proximal tubular cells to glucose leads to accumulation of type IV collagen and fibronectin by decreased degradation. Kidney Int 52: 973-984.

33. Shan SW, Lee DY, Deng Z, Shatseva T, Jeyapalan Z, et al. (2009) MicroRNA MiR-17 retards tissue growth and represses fibronectin expression. Nat Cell Biol 11: 1031-1038. doi:10.1038/ncb1917.

34. Hackl M, Brunner S, Fortschegger K, Schreiner C, Micutkova L, et al. (2010) miR-17, miR-19b, miR-20a, and miR-106a are down-regulated in human aging. Aging Cell 9: 291-296. doi:10.1111/j.1474-9726.2010.00549.x.

35. Sun H, Li Q-W, Lv X-Y, Ai J-Z, Yang Q-T, et al. (2010) MicroRNA-17 posttranscriptionally regulates polycystic kidney disease-2 gene and promotes cell proliferation. Mol Biol Rep 37: 2951-2958. doi:10.1007/s11033-009-9861-3.

36. Tran U, Zakin L, Schweickert A, Agrawal R, Döger R, et al. (2010) The RNAbinding protein bicaudal $\mathrm{C}$ regulates polycystin 2 in the kidney by antagonizing miR-17 activity. Development 137: 1107-1116. doi:10.1242/dev.046045.

37. Hou J, Shan Q, Wang T, Gomes AS, Yan Q et al. (2007) Transgenic RNAi Depletion of Claudin-16 and the Renal Handling of Magnesium. Journal of Biological Chemistry 282: 17114-17122. doi:10.1074/jbc.M700632200.

38. Mahimainathan L, Das F, Venkatesan B, Choudhury GG (2006) Mesangial cell hypertrophy by high glucose is mediated by downregulation of the tumor suppressor PTEN. Diabetes 55: 2115-2125. doi:10.2337/db05-1326.

39. Chun-Zhi Z, Lei H, An-Ling Z, Yan-Chao F, Xiao Y, et al. (2010) MicroRNA221 and microRNA-222 regulate gastric carcinoma cell proliferation and radioresistance by targeting PTEN. BMC Cancer 10: 367. doi:10.1186/14712407-10-367.

40. Fatima S, Zhou S, Sorrentino BP (2012) Abcg2 expression marks tissue-specific stem cells in multiple organs in a mouse progeny tracking model. Stem Cells 30: 210-221. doi:10.1002/stem.1002.

41. Liao R, Sun J, Zhang L, Lou G, Chen M, et al. (2008) MicroRNAs play a role in the development of human hematopoietic stem cells. J Cell Biochem 104: 805817. doi:10.1002/jcb.21668.

42. Valera VA, Walter BA, Linehan WM, Merino MJ (2011) Regulatory Effects of microRNA-92 (miR-92) on VHL Gene Expression and the Hypoxic Activation of miR-210 in Clear Cell Renal Cell Carcinoma. J Cancer 2: 515-526.

43. Dentelli P, Rosso A, Orso F, Olgasi C, Taverna D, et al. (2010) microRNA-222 controls neovascularization by regulating signal transducer and activator of transcription 5A expression. Arterioscler Thromb Vasc Biol 30: 1562-1568. doi:10.1161/ATVBAHA.110.206201.

44. Togliatto G, Trombetta A, Dentelli P, Rosso A, Brizzi MF (2011) MIR221/ MIR222-driven post-transcriptional regulation of P27KIP1 and P57KIP2 is crucial for high-glucose- and AGE-mediated vascular cell damage. Diabetologia 54: 1930-1940. doi:10.1007/s00125-011-2125-5.
45. Ghosh G, Subramanian IV, Adhikari N, Zhang X, Joshi HP, et al. (2010) Hypoxia-induced microRNA-424 expression in human endothelial cells regulates HIF- $\alpha$ isoforms and promotes angiogenesis. J Clin Invest 120: 41414154. doi:10.1172/JCI42980.

46. Chamorro-Jorganes A, Araldi E, Penalva LOF, Sandhu D, FernándezHernando C, et al. (2011) MicroRNA-16 and MicroRNA-424 Regulate CellAutonomous Angiogenic Functions in Endothelial Cells via Targeting Vascular Endothelial Growth Factor Receptor-2 and Fibroblast Growth Factor Receptor1. Arteriosclerosis, Thrombosis, and Vascular Biology. Available:http://www. ncbi.nlm.nih.gov/pubmed/21885851. Accessed 25 September 2011.

47. Kato M, Zhang J, Wang M, Lanting L, Yuan H, et al. (2007) MicroRNA-192 in diabetic kidney glomeruli and its function in TGF-beta-induced collagen expression via inhibition of E-box repressors. Proc Natl Acad Sci USA 104: 3432-3437. doi:10.1073/pnas.0611192104.

48. Kato M, Arce L, Wang M, Putta S, Lanting L, et al. (2011) A microRNA circuit mediates transforming growth factor- $\beta 1$ autoregulation in renal glomerular mesangial cells. Kidney Int 80: 358-368. doi:10.1038/ki.2011.43.

49. Krupa A, Jenkins R, Luo DD, Lewis A, Phillips A, et al. (2010) Loss of MicroRNA-192 Promotes Fibrogenesis in Diabetic Nephropathy. Journal of the American Society of Nephrology 21: 438-447. doi:10.1681/ASN.2009050530.

50. Macconi D, Tomasoni S, Romagnani P, Trionfini P, Sangalli F, et al. (2012) MicroRNA-324-3p Promotes Renal Fibrosis and Is a Target of ACE Inhibition. JASN 23: 1496-1505. doi:10.1681/ASN.2011121144.

51. García-Sánchez O, López-Hernández FJ, López-Novoa JM (2010) An integrative view on the role of TGF-beta in the progressive tubular deletion associated with chronic kidney disease. Kidney Int 77: 950-955. doi:10.1038/ ki.2010.88.

52. Ostendorf T, Eitner F, Floege J (2011) The PDGF family in renal fibrosis. Pediatr Nephrol. Available:http://www.ncbi.nlm.nih.gov/pubmed/21597969. Accessed 4 September 2011.

53. Titan SM, Zatz R, Graciolli FG, dos Reis LM, Barros RT, et al. (2011) FGF-23 as a Predictor of Renal Outcome in Diabetic Nephropathy. Clinical Journal of the American Society of Nephrology 6: 241-247. doi:10.2215/CJN.04250510.

54. Heese K, Beck K-F, Behrens MH, Plüss K, Fierlbeck W, et al. (2003) Effects of high glucose on cytokine-induced nerve growth factor (NGF) expression in rat renal mesangial cells. Biochem Pharmacol 65: 293-301.

55. Micera A, Vigneti E, Pickholtz D, Reich R, Pappo O, et al. (2001) Nerve growth factor displays stimulatory effects on human skin and lung fibroblasts, demonstrating a direct role for this factor in tissue repair. Proc Natl Acad Sci USA 98: 6162-6167. doi:10.1073/pnas.101130898.

56. Bonofiglio R, Antonucci MT, Papalia T, Romeo F, Capocasale G, et al. (2007) Nerve growth factor (NGF) and NGF-receptor expression in diseased human kidneys. J Nephrol 20: 186-195.

57. Shan D, Wu HM, Yuan QY, Li J, Zhou RL, et al. (2012) Pentoxifylline for diabetic kidney disease. Cochrane Database Syst Rev 2: CD006800. doi:10.1002/14651858.CD006800.pub2

58. Sharma K, Ix JH, Mathew AV, Cho M, Pflueger A, et al. (2011) Pirfenidone for diabetic nephropathy. J Am Soc Nephrol 22: 1144-1151. doi:10.1681/ ASN.2010101049.

59. Pergola PE, Raskin P, Toto RD, Meyer CJ, Huff JW, et al. (2011) Bardoxolone methyl and kidney function in CKD with type 2 diabetes. N Engl J Med 365: 327-336. doi:10.1056/NEJMoa 1105351

60. Ruggenenti P, Fassi A, Ilieva AP, Bruno S, Iliev IP, et al. (2004) Preventing microalbuminuria in type 2 diabetes. $N$ Engl J Med 351: 1941-1951. doi:10.1056/NEJMoa042167.

61. Haller H, Ito S, Izzo JL Jr, Januszewicz A, Katayama S, et al. (2011) Olmesartan for the delay or prevention of microalbuminuria in type 2 diabetes. N Engl J Med 364: 907-917. doi:10.1056/NEJMoa1007994.

62. Scian MJ, Maluf DG, David KG, Archer KJ, Suh JL, et al. (2011) MicroRNA Profiles in Allograft Tissues and Paired Urines Associate With Chronic Allograft Dysfunction With IF/TA. American Journal of Transplantation: Official Journal of the American Society of Transplantation and the American Society of Transplant Surgeons. Available:http://www.ncbi.nlm.nih.gov/pubmed/ 21794090 . Accessed 25 September 2011.

63. Neal CS, Michael MZ, Pimlott LK, Yong TY, Li JYZ, et al. (2011) Circulating microRNA expression is reduced in chronic kidney disease. Nephrology, Dialysis, Transplantation: Official Publication of the European Dialysis and Transplant Association - European Renal Association. Available:http://www. ncbi.nlm.nih.gov/pubmed/21891774. Accessed 25 September 2011.

64. Weber JA, Baxter DH, Zhang S, Huang DY, Huang KH, et al. (2010) The microRNA spectrum in 12 body fluids. Clin Chem 56: 1733-1741. doi:10.1373/ clinchem.2010.147405. 